\title{
Q1 Substrato vegetal utilizado para nidificação de vespas sociais (Hymenoptera, Vespidae) em Floresta Decidual
}

Gabriela dos Santos Francisco ${ }^{1}$

Marcos Magalhães de Souza²

Mateus Clemente ${ }^{3}$

Ângela Gomes Brunismann

\section{Resumo}

As vespas sociais nidificam em diferentes espécies de plantas, no entanto, as informações em relação à possível preferência por determinados tipos de substratos vegetais são escassas em diferentes ecossistemas brasileiros. Nesse panorama, o presente estudo tem por objetivo obter informações sobre nidificação desses insetos em áreas de Floresta Decidual. 0 trabalho foi realizado no estado de Minas Gerais, Brasil, no período de junho de 2014 a abril de 2015. Utilizando a metodologia de busca ativa, foram registradas 128 colônias de 18 espécies distribuídas em 12 gêneros de vespas sociais nidificadas em 30 famílias vegetais. A escolha das vespas sociais pelo substrato de nidificação é influenciada pela complexidade ecológica do ambiente.

Palavras-chave: Colônias. Insetos sociais. Mata seca.

\section{Introdução}

O Brasil é caracterizado por formações florestais distintas, e $6 \%$ da cobertura vegetal brasileira é representada por Floresta Decidual, conhecida popularmente como Mata Seca (SEVILHA; SCARIOT; NORONHA, 2004). O norte do Estado de Minas Gerais recebe a influência da Floresta Decidual, que ocupa uma área de 3,46 \% (BELEM, 2008), formando um ecótono entre Caatinga e Cerrado (SEVILHA; SCARIOT; NORONHA, 2004), tendo como características marcantes o alto grau de caducifólia na estação seca (MURPHY; LUGO, 1986) e duas estações climáticas bem definidas (VELOSO; RANGEL-FILHO; LIMA, 1991; COLLEVATTI et al., 2013).

Apesar de essa formação não ocupar uma área geográfica considerável no estado de Minas Gerais, os poucos trabalhos realizados sobre a diversidade apontam uma relevante riqueza de determinados grupos de insetos (OLIVEIRA et al., 2011) e plantas (SALES et al., 2009 a,b), contudo não há dados sobre vespas sociais.

\footnotetext{
1 Instituto Federal de Educação, Ciência e Tecnologia do Sul de Minas Gerais (IFSULDEMINAS), Campus Inconfidentes, Minas Gerais, Brasil, graduado em Licenciatura em Ciências Biológicas, gabifransantos2013@gmail.com, Rua Humaitá, 1038, Bairro Várzea, Ouro Fino (MG), CEP: 37570-000.

2 Instituto Federal de Educação, Ciência e Tecnologia do Sul de Minas Gerais (IFSULDEMINAS), Campus Inconfidentes, professor, marcos.souza@ifsuldeminas.edu.br.

3 Universidade Estadual Paulista "Júlio de Mesquita Filho", Departamento de Zoologia, Instituto de Biociências, professor, mateus1981@gmail.com.

4 Instituto Federal de Educação, Ciência e Tecnologia do Sul de Minas Gerais (IFSULDEMINAS), Campus Inconfidentes, graduada em Licenciatura em Ciências Biológicas, angelabrunismann@gmail.com.
} 
As vespas sociais pertencem à ordem Hymenoptera, conhecidas popularmente como marimbondos ou cabas (PREZOTO; CORTES; MELO, 2008), e estão inseridas na subfamília Polistinae. No Brasil são encontradas 319 espécies sendo 104 endêmicas, é o país com a maior diversidade de Polistinae do mundo (CARPENTER; ANDENA, 2013). A Subfamília está dividida em três tribos: Mischocyttarini (Mischocyttarus 117 espécies), Polistini (Polistes 38 espécies) e Epiponini (20 gêneros e 149 espécies) (CARPENTER; ANDENA, 2013). O grupo Epiponini possui a maior riqueza de gêneros de vespas sociais (CARPENTER; ANDENA, 2013) e uma grande variedade de arquitetura dos ninhos (WENZEL, 1998).

A permanência das vespas sociais em determinados ambientes depende diretamente do sucesso da construção de novas colônias (DEJEAN; CORDABA; CARPENTER, 1998; HUNT, 2007; JEANNE, 1975), que envolvem a grande capacidade de forrageamento, cujo objetivo é buscar alimento e material para a fundação dos ninhos envolvendo a coleta dos seguintes recursos: carboidratos utilizados na alimentação de adultos, proteínas animal utilizada na alimentação dos imaturos, fibra vegetal utilizada como material de construção dos ninhos e água utilizada para a refrigeração e como material de construção dos ninhos (PREZOTO; CORTES; MELO, 2008; ELISEl et al., 2010; BARBOSA; PASCHOALINI; PREZOTO, 2014).

Os substratos utilizados para fundação de suas colônias são bem variados, podendo ser superfície inferior de folhas, edificações, troncos de árvores, ramos vegetais ou cavidades naturais (CARPENTER; ANDENA, 2013) e seus ninhos apresentam diversas variedades quanto à arquitetura, baseada no tipo de substrato utilizado (JEANNE, 1975; WENZEL, 1998), podendo ter um único favo coberto ou não por um envelope protetor ou múltiplos favos também cobertos ou descobertos, sendo ainda utilizados como caráter taxonômico para as espécies (CARPENTER; ANDENA, 2013). Entretanto, não está claro quais são os fatores que levam à escolha de um determinado substrato vegetal em relação a outro (SOUZA; PREZOTO, 2006; PREZOTO et al., 2007; SOMAVILLA; OLIVEIRA; SILVEIRA, 2012; SOUZA et al., 2014).

A partir do que foi apresentado, o presente estudo visou obter mais informações sobre os substratos vegetais utilizados para a nidificação das vespas sociais em floresta decidual.

\section{Material e métodos}

O trabalho foi conduzido no norte do Estado de Minas Gerais em um ambiente que recebe a influência dos biomas Cerrado e Caatinga. A região possui condições climáticas que refletem um clima semiárido, tendo uma temperatura média por ano de $25^{\circ} \mathrm{C}$ e índices pluviométricos anuais de aproximadamente $1.000 \mathrm{~mm}$, com chuvas concentradas especialmente nos meses de outubro a fevereiro (SANTOS et al., 2007). 
Figura 01: Áreas amostradas, hachuradas no mapa, para registro das colônias de vespas sociais em Minas Gerais.

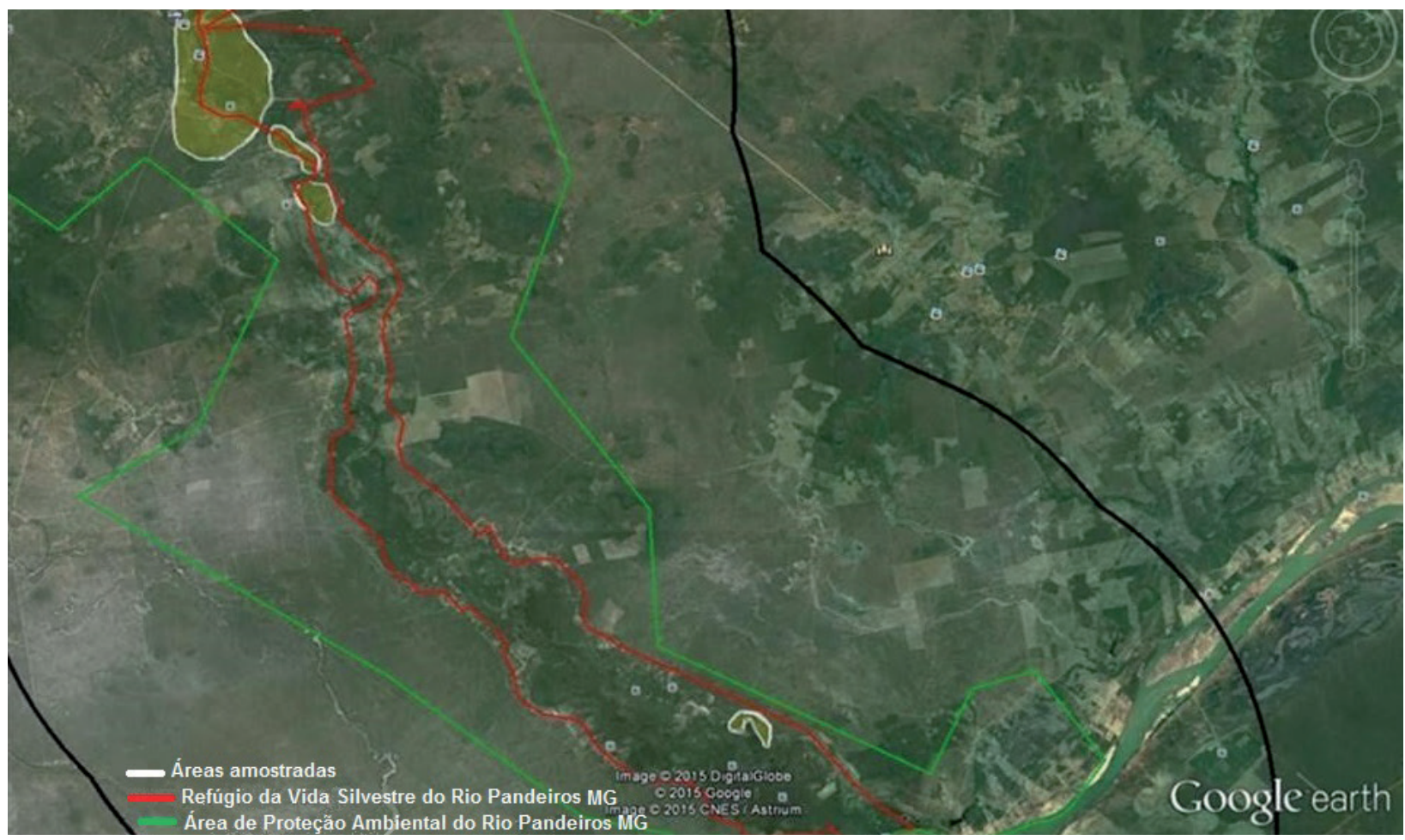

Fonte: Elaboração dos autores (2016)

As coletas foram realizadas no período de junho de 2014 a abril de 2015, sendo cinco dias de amostragem em cada estação do ano. Foram quatro períodos amostrais: estação chuvosa (primavera e verão) e estação seca (outono e inverno), totalizando 20 dias de coleta no período de um ano, quando foram amostradas $10 \%$ da área.

Para o registro das colônias de vespas sociais nidificadas em substrato vegetal foi utilizado o método de busca ativa. A coleta de espécimes de vespas sociais foi feita com redes entomológicas. Os gêneros foram identificados com base em chaves propostas por Richards (1978).

O material vegetal utilizado como substrato foi coletado com o auxílio de uma tesoura de poda ou podão, sendo prensado e posteriormente levado até a estufa do Laboratório de Sementes do Campus Inconfidentes para secagem. Quando secas, foram montadas exsicatas das plantas para identificação. A identificação do material vegetal foi realizada com o auxílio da literatura especializada Botânica Sistemática (APG III 2012) e por especialistas da área.

\section{Resultados e discussão}

Foram registradas 128 colônias de 18 espécies distribuídas em 12 gêneros de vespas sociais (TABELA 01). As espécies com o maior número de colônias foram Polybia occidentalis occidentalis (OLIVER, 1971) com 33; Mischocyttarus montei (ZIKAN, 1949) com 19 colônias; Protopolybia exigua exigua (SAUSSURE, 1854) com 18 e Mischocyttarus cassununga (R. VON. IHERING, 1903) com 15 colônias. O restante das espécies de vespas sociais apresentaram um número inferior a 10 colônias nidificadas em substrato vegetal (TABELA 01). 
Tabela 01. Relação das espécies de vespas sociais, número de colônias, espécies vegetais e famílias das plantas que foram utilizadas como substrato de nidificação, registrados no período de junho de 2014 a abril de 2015.

\begin{tabular}{|c|c|c|c|}
\hline Espécies de vespas sociais & Espécies vegetais & Famílias & Colônias \\
\hline $\begin{array}{l}\text { Agelaia multipicta multipicta } \\
\text { (HALIDAY, 1836) }\end{array}$ & sp. 01 & Sapindaceae & 1 \\
\hline $\begin{array}{l}\text { Agelaia vicina } \\
\text { (SAUSSURE, 1854) }\end{array}$ & Hymenaea courbaril L. & Fabaceae & 1 \\
\hline \multirow{2}{*}{$\begin{array}{l}\text { Apoica pallens } \\
\text { (FABRICIUS, 1804) }\end{array}$} & sp. 01 & Myrtaceae & 1 \\
\hline & Mangifera indica L. & Anacardiaceae & 1 \\
\hline $\begin{array}{l}\text { Brachygastra lecheguana } \\
\text { (LATREILLE, 1824) }\end{array}$ & Mangifera indica L. & Anacardiaceae & 1 \\
\hline \multirow{3}{*}{$\begin{array}{l}\text { Chartergellus communis } \\
\text { (RICHARDS, 1978) }\end{array}$} & Cedrela fissilis Vell. & Meliaceae & 1 \\
\hline & Citrus sp. L. & Rutaceae & 1 \\
\hline & Mangifera indica L. & Anacardiaceae & 1 \\
\hline $\begin{array}{l}\text { Chartergus globiventris } \\
\text { (SAUSSURE, 1854) }\end{array}$ & sp. 01 & Fabaceae & 2 \\
\hline \multirow{2}{*}{$\begin{array}{l}\text { Metapolybia cingulata } \\
\text { (FABRICIUS, 1804) }\end{array}$} & $\begin{array}{l}\text { Hymenaea stigonocarpa Mart. } \\
\text { ex Hayne }\end{array}$ & Fabaceae & 3 \\
\hline & sp.02 & Fabaceae & 1 \\
\hline \multirow{4}{*}{$\begin{array}{l}\text { Mischocyttarus bertonii } \\
\text { (DUCKE, 1918) }\end{array}$} & sp.03 & Fabacea & 2 \\
\hline & sp.01 & Poaceae & 1 \\
\hline & sp.01 & Meliaceae & 1 \\
\hline & Mangifera indica L. & Anacardiaceae & 2 \\
\hline \multirow{8}{*}{$\begin{array}{l}\text { Mischocyttarus cassununga } \\
\text { (R. VON IHERING, 1903) }\end{array}$} & Davilla sp. & Dilleneaceae & 1 \\
\hline & Nephrolepis exaltata & Pteridófita & 2 \\
\hline & Mangifera indica L. & Anacardiaceae & 5 \\
\hline & Anacardium occidentale L. & Anacardiaceae & 2 \\
\hline & sp.01 & Bignoniaceae & 1 \\
\hline & sp.01 & Solanaceae & 1 \\
\hline & Peperomia sp. & Piperaceae & 1 \\
\hline & Solanum pseudo-quina & Solanaceae & 2 \\
\hline \multirow{7}{*}{$\begin{array}{l}\text { Mischocyttarus montei } \\
\text { (ZIKAN, 1949) }\end{array}$} & sp.01 & Delineaceae & 1 \\
\hline & Mangifera indica L. & Anacardiaceae & 8 \\
\hline & Anacardium occidentale L. & Anacardiaceae & 1 \\
\hline & Inga vera Willd. & Fabaceae & 2 \\
\hline & Caryocar brasiliense Cambess. & Caryocaraceae & 1 \\
\hline & Coccoloba sp. & Polygonaceae & 2 \\
\hline & Mangifera indica L. & Anacardiaceae & 4 \\
\hline \multirow{2}{*}{$\begin{array}{l}\text { Parachartergus fraternus } \\
\text { (GRIBOLDO, 1892) }\end{array}$} & Celtis sp. & Cannabaceae & 1 \\
\hline & Hymenaea courbaril L. & Fabaceae & 1 \\
\hline \multirow{2}{*}{$\begin{array}{l}\text { Parachartergus smithii } \\
\text { (SAUSSURE, 1854) }\end{array}$} & $\begin{array}{l}\text { Anadenanthera colubrina(Vell.) } \\
\text { Brenam }\end{array}$ & Fabaceae & 1 \\
\hline & sp.04 & Fabaceae & 1 \\
\hline
\end{tabular}




\begin{tabular}{|c|c|c|c|}
\hline \multicolumn{4}{|l|}{ (Continuação) } \\
\hline Espécies de vespas sociais & Espécies vegetais & Famílias & Colônias \\
\hline \multirow{7}{*}{$\begin{array}{l}\text { Polybia jurinei } \\
\text { (SAUSSURE, 1854) }\end{array}$} & sp.01 & Bursemaceae & 1 \\
\hline & sp.05 & Fabaceae & 1 \\
\hline & Prunus cf. myrtilolia L. & Rosaceae & 1 \\
\hline & sp.06 & Fabaceae & 2 \\
\hline & sp.02 & Bignoniaceae & 1 \\
\hline & sp.01 & Sapotaceae & 1 \\
\hline & sp.01 & Rubiaceae & 1 \\
\hline \multirow{28}{*}{$\begin{array}{l}\text { Polybia occidentalis occidentalis } \\
\text { (OLIVIER, 1791) }\end{array}$} & cipo sp. & Poaceae & 1 \\
\hline & Platypodium elegans Vog. & Fabaceae & 2 \\
\hline & sp.07 & Fabaceae & 1 \\
\hline & Pitoge nienitins & Fabaceae & 1 \\
\hline & Bauhinia forficata Vog. & Fabaceae & 1 \\
\hline & sp.08 & Fabaceae & 1 \\
\hline & Hymenaea courbaril L. & Fabaceae & 4 \\
\hline & sp.09 & Fabaceae & 1 \\
\hline & sp. 10 & Fabaceae & 1 \\
\hline & sp.11 & Fabaceae & 1 \\
\hline & Aquenia & Fabaceae & 1 \\
\hline & sp.01 & Ericaceae & 1 \\
\hline & Anacardium occidentale L. & Anacardiaceae & 1 \\
\hline & sp.01 & Anacardiaceae & 1 \\
\hline & Ficus sp.01 & Moraceae & 1 \\
\hline & sp.01 & Moraceae & 1 \\
\hline & Celtis sp. & Cannabaceae & 1 \\
\hline & Persea americana Mill. & Lauraceae & 1 \\
\hline & sp.01 & Lauraceae & 1 \\
\hline & Terminalia catappa L. & Combretaceae & 1 \\
\hline & sp.01 & Santidaceae & 1 \\
\hline & Pisidium guajava L. & Myrtaceae & 2 \\
\hline & sp.01 & Malpighiaceae & 1 \\
\hline & sp.01 & Werbenaceae & 1 \\
\hline & sp.01 & Nytaginaceae & 1 \\
\hline & sp.01 & Ulmaceae & 1 \\
\hline & sp.01 & Anonaceae & 1 \\
\hline & Caryocar brsiliense St. Hil & Caryocaraceae & 1 \\
\hline \multirow{3}{*}{$\begin{array}{l}\text { Protonectarina sy/veirae } \\
\text { (SAUSSURE, 1854) }\end{array}$} & sp.02 & Moraceae & 1 \\
\hline & sp.02 & Myrtaceae & 1 \\
\hline & sp.02 & Anacardiaceae & 1 \\
\hline
\end{tabular}




\begin{tabular}{|c|c|c|c|}
\hline \multicolumn{4}{|l|}{ (Continuação) } \\
\hline Espécies de vespas sociais & Espécies vegetais & Famílias & Colônias \\
\hline \multirow{11}{*}{$\begin{array}{l}\text { Protopolybia exigua exigua } \\
\text { (SAUSSURE, 1854) }\end{array}$} & Anacardium occidentale L. & Anacardiaceae & 2 \\
\hline & Mangifera indica L. & Anacardiaceae & 5 \\
\hline & Eugenia sp. & Myrtaceae & 1 \\
\hline & sp.03 & Myrtaceae & 1 \\
\hline & Xylopia aromatica (Lam.) Mart. & Annonaceae & 1 \\
\hline & Macherium sp. & Fabaceae & 1 \\
\hline & Pylopia aromatica (Lam.) & Anonaceae & 1 \\
\hline & Talisia esculenta St. Hil & Sapindaceae & 1 \\
\hline & Ficus sp.02 & Moraceae & 1 \\
\hline & Terminalia catappa L. & Combretaceae & 2 \\
\hline & Caryocar brsiliense St. Hil & Caryocaraceae & 2 \\
\hline Protopolybia sedula (SAUSSURE, 1854) & Hymenaea courbaril L. & Fabaceae & 1 \\
\hline \multirow{5}{*}{ Synoeca surinama (LINNAEUS, 1767) } & $\begin{array}{l}\text { Hymenaea stigonocarpa Mart. } \\
\text { ex Hayne }\end{array}$ & Fabaceae & 2 \\
\hline & Inga vera Willd. & Fabaceae & 2 \\
\hline & sp.04 & Myrtaceae & 1 \\
\hline & Ficus sp.03 & Moraceae & 1 \\
\hline & sp.01 & Rutaceae & 1 \\
\hline Total & 71 Espécies vegetais & 30 & 128 \\
\hline
\end{tabular}

Fonte: Elaboração dos autores (2016)

No total foram identificadas 30 famílias vegetais, sendo 29 angiospermas e uma pteridófita que foram utilizadas como substrato para nidificação. As famílias que apresentaram maior número de espécies vegetais também foram as mais utilizadas como substrato de nidificação pelos insetos sociais. Fabaceae com 37 (28,90 \%) colônias em 19 espécies vegetais e Anacardiaceae com 35 (27,34 \%) colônias em quatro espécies de plantas (TABELA 01).

O levantamento florístico realizado por Rodrigues et al. (2009) na mesma região aponta Fabaceae, Myrtaceae e Anacardiaceae como as famílias que apresentaram maior riqueza de espécies, sendo comum em formações florestais adjacentes, o que aumenta a probabilidade das vespas sociais utilizarem essas espécies vegetais como substratos para nidificação.

A espécie vegetal mais utilizada como substrato de nidificação foi Mangifera indica L. (27 colônias), popularmente conhecida como mangueira, da família Anacardiaceae. A espécie apresenta folha larga e rígida e isso poderia influenciar positivamente a edificação de colônias de vespas sociais, em razão da proteção contra intempéries.

A escolha das vespas sociais por um substrato de nidificação geralmente é influenciada por plantas com folhas largas e perenes (DEJEAN; CORDABA; CARPENTER, 1998; SOUZA et al., 2014). Essas plantas contribuem de maneira significativa para as vespas sociais, fornecendo sombra para as colônias durante o período severo do ano. Alguns estudos afirmam que espécies do gênero Mischocyttarus tem preferência por ambientes antropizados, principalmente locais que protegem os ninhos da chuva e dos raios solares (SIMÕES; GOBBI; BATARCE, 1985; LIMA; LIMA; PREZOTO, 2000; TORRES et al., 2011) isso explica a preferência do gênero por espécies vegetais que ofereçam proteção contra alguns fatores externos. 
As características morfológicas das plantas não foram analisadas no presente estudo, mas podem ser um fator que contribua para a escolha das vespas sociais por um substrato de nidificação, principalmente na escolha do gênero Mischocyttarus, que constrói seus ninhos abertos e por isso necessitam de mais proteção contra fatores abióticos e bióticos.

As espécies Hymenaea coubaril L. e Hymenaea stgonocarpa Mart.ex Hayne foram bastante representativas, a $H$. coubaril L. apresentou 12 colônias e a H. stgonocarpa Mart.exHayn cinco colônias.

Hymenaea stgonocarpa tem ocorrência nos estados do Piauí, Bahia, Goiás, Minas Gerais, Mato Grosso do Sul e São Paulo, podendo ser encontrada no Cerrado por esse motivo, também é conhecida como jatobá-do-cerrado (CORRÊA, 1984; LORENZI, 2000), é uma árvore decídua, com folhas alternadas compostas bifoliadas e tronco tortuoso característico de planta do Cerrado (EMPRESA BRASILEIRA DE PESQUISA AGROPECUÁRIA- EMPRAPA, 2010). A espécie H. coubaril L. pode ser encontrada em áreas de estação seca como a Bahia, tendo ocorrência em formações abertas da Caatinga e Cerrados (LORENZI, 2000; PINTO; SANTIAGO; LAMEIRA, 2000; LORENZI), suas folhas são compostas e coriáceas, o que também explicaria as vespas sociais utilizarem esta espécie para realizar a nidificação em suas folhas.

A espécie de vespa social que apresentou a maior plasticidade em relação ao substrato de nidificação foi Polybia occidentalis com 33 colônias. A nidificação ocorreu em diferentes famílias vegetais, com destaque para Fabaceae (14 colônias), para a qual o gênero Hymenaea e especificamente a espécie Hymenaea courbaril L. foi a mais utilizada pela espécie $P$. occidentalis com quatro colônias.

A possível preferência das vespas sociais pelo substrato de nidificação, devido a alguma característica morfológica ou fisiológica de alguma espécie vegetal em particular, ainda não está clara (SOUZA; PREZOTO, 2006; PREZOTO et al., 2007; SOUZA et al., 2010; SOMAVILLA et al., 2012; SOUZA et al., 2014), pois ainda há poucos estudos que tentam explicar uma possível preferência de algumas espécies de vespas sociais por determinadas espécies de plantas, entretanto, o trabalho realizado por Souza et al., 2014 mostra que as vespas não são influenciadas pela particularidade de uma espécie vegetal em específico, e sim por um atributo compartilhado por várias espécies vegetais, como folhas largas e coriáceas, deciduidade, entre outras características. Assim, a complexidade do ambiente é um fator significativo na seleção do local de nidificação (DEJEAN; CORDABA; CARPENTER, 1998).

\section{Conclusão}

Foi possível constatar que as vespas sociais em Floresta Decidual não são influenciadas por alguma característica específica das espécies vegetais, e sim pela riqueza de algumas famílias. Neste estudo, Fabaceae e Anacardiaceae foram as mais abundantes na área de estudo e as mais utilizadas como substrato de nidificação, sendo que a complexidade ecológica do ambiente é determinante na escolha pelo substrato vegetal, corroborando os resultados obtidos por outros estudos realizados em ecossistemas distintos. 


\title{
Vegetatives ubstrateused for nesting of social wasps (Hymenoptera, Vespidae) in Decidual Forest
}

\begin{abstract}
Social wasps nest in different plant species, however, information regarding the possible preference for certain types of plant substrates is scarce in different Brazilian ecosystems. In this panorama, the present study aims to obtain information on nesting of these insects in Deciduous Forest areas. The work was carried out in the state of Minas Gerais, Brazil, from June 2014 to April 2015. Using the active search methodology, 128 colonies of 18 species distributed in 12 genera of nested wasps in 30 plant families were registered. The choice of social wasps by the nesting substrate is influenced by the ecological complexity of the environment.
\end{abstract}

Keywords: Colonies. Social insects. Dryforest.

\section{Referências}

BARBOSA, B. C.; PASCHOALINI, M.; PREZOTO, F. Temporal Activity Patterns and Foraging Behaviour by Social Wasps (Hymenoptera, Polistinae) on Fruits of Mangifera indica L. (Anacardiaceae). Sociobiology, v. 61, p. 239-242, 2014. Disponível em: <http://periodicos.uefs.br/index.php/sociobiology/ article/view/253/422 >. Acesso em: 16 set. 2018. DOI: 10.13102/sociobiology.v61i2.239-242.

BELEM, R. A. Zoneamento ambiental e os desafios da implementação do Parque Estadual Mata Seca, Município de Manga, Norte de Minas Gerais. 2008. 169 f. Tese (Mestrado) UFMG. Disponível em: <http://www.bibliotecadigital.ufmg.br/dspace/handle/1843/MPBB-7FCMWK>. Acesso em: 16 set. 2018.

CARPENTER, J. M.; ANDENA, S. R. The vespidae of Brazil. Manaus, Instituto Nacional de Pesquisa da Amazônia, p. 42, 2013.

CARPENTER, J. M.; MARQUES, O. M. Contribuição ao estudo de vespídeos do Brasil (Insecta, Hymenoptera, Vespoidea, Vespidae). Cruz das Almas: Universidade Federal da Bahia, 2001. (Série Publicações Digitais. v. 2), versão 1.0. 1 CDROM.

COLLEVATTI, R. G; TERRIBILE, L. C; OLIVEIRA, G.; LIMA RIBEIRO, M. S; NABOUT, J. C; RANGEL, T. F.; DINIZ-FILHO, J. A. F. Drawbacks to palaeodistribution modelling: the case of South American seasonally dry forests. Journal of Biogeography. v. 40 , n. 2, p. 345-358, 2013. Disponível em: http://dx.doi.org/10.1111/jbi.12005. Acesso em: 16 set. 2018.

CORRÊA, M. P. Dicionário das plantas úteis do Brasil e das exóticas cultivadas. Rio de Janeiro. Ministério da Agricultura: Instituto Brasileiro de Desenvolvimento Florestal, 1984. p. 707.

DEJEAN, A.; CORDABA, B.; CARPENTER, J. M. Nesting site selection by wasp in the Guianese rainforest. Insectes sociaux, v. 45, p. 33-41, 1998. Disponível em: <https://link.springer.com/ article/10.1007/s000400050066> . Acesso em: 16 set. 2018. DOl: https://doi.org/10.1007/ s000400050066 
ELISEI, T.; NUNES, J. V. E.; RIBEIRO JUNIOR, C.; FERNANDES JUNIOR, A. J.; PREZOTO, F. Uso da vespa social Polybia versicolor no controle de desfolhadores de eucalipto. Pesquisa Agropecuária Brasileira. 2010, v. 45, p. 958-964. Disponível em: <http://seer.sct.embrapa.br/index.php/pab/article/view/8202/6060 >. Acesso em: 16 set. 2018.

EMBRAPA. Cultivo da Mangueira. Semiárido Sistemas de Produção, 2. ed., versão eletrônica, p. 111, 2010.

HUNT, J. H. The Evolution of Social Wasps. Oxford University Press, New York, v. 259, 2007. p. 100-114.

JEANNE, R. L. The adaptiveness of social wasps nest architecture. The Quarterly Review of Biology, v. 50, p. 267, 1975. Disponível em: <https://www.journals.uchicago.edu/doi/10.1086/408564>. Acesso em: 19 set. 2018. DOI: https://doi.org/10.1086/408564

LIMA, M. A. P.; LIMA, J. R. D.; PREZOTO, F. Levantamento dos gêneros, flutuação das colônias e hábitos de nidificação de vespas sociais (Hymenoptera, Vespidae) no Campus da UFJF, Juiz de Fora, MG. Revista Brasileira de Zoociências, 2: 69-80, 2000.

LORENZI, H. Árvores brasileiras: manual de identificação e cultivo de plantas arbóreas nativas do Brasil. São Paulo: Plantarum, 2000. p.352.

LORENZI, H.; MATOS, F. J. A. Plantas medicinais no Brasil: nativas e exóticas. São Paulo: Plantarum, 2002. p. 512.

MURPHY, P. G.; LUGO, A. E. Ecology of tropical dry forest. Annual Review of Ecology and Systematics, v. 17 , n. 1 , p. $67-88,1986$.

OLIVEIRA, V. H. F.; MOTA-SOUZA, J. G.; VAZ-DE-MELLO, F. Z.; NEVES, F. S.; FAGUNDES, M. Variação na fauna de besouros rola-bosta (Coleoptera: Scarabaeinae) 24 entre habitats de Cerrado, Mata Seca e Mata Ciliar em uma região de transição Cerrado-Caatinga no Norte de Minas Gerais MG. Biota, v. 4, p. 4-16, 2011.

PINTO, J. E. B. P.; SANTIAGO, E. J. A.; LAMEIRA, O. A. Compêndio de plantas medicinais. Lavras: UFLA/FAEPE, p. 208, 2000.

PREZOTO, F.; RIBEIRO-JÚNIOR, C.; OLIVEIRA, S. A.; ELISEI, T. Manejo de vespas e marimbondos em ambiente urbano. In: PINTO, A. S.; ROSSI, M. M.; SALMERON, E. (org.). Manejo de Pragas Urbanas. Piracicaba: editor, 2007. p. 123-126.

PREZOTO, F.; CORTES, S. A. O.; MELO, A. C. Vespas: de vilãs a parceiras. Ciências Hoje, v. 48, p. 70-73, 2008.

RICHARDS, O. W. The social Wasps of the Americas, Excluding the Vespinae. London: British Museum, Natural History, 1978. p. 580. 
RODRIGUES, P. M. S.; AZEVEDO, I. F. P.; VElOSO, M. D. M.; SANTOS, R. M.; MENINO, G. C. O.; NUNES, R. F.; FERNANDES, W. Riqueza Florística da vegetação ciliar do Rio Pandeiros, Norte de Minas Gerais, MG. Biota, v. 2, n. 2, p. 20 - 37, 2009. Disponível em: <https://documentacao.socioambiental.org/noticias/anexo_noticia//6583_20091113_120335.pdf\#page $=18>$. Acesso em: 17 set. 2018.

SALES, H. R.; SANTOS, R. M. dos; NUNES, Y. R. F.; MORAIS-COSTA, F.; SOUZA, S. C. A. Caracterização florística de um fragmento de cerrado na APA Estadual do Rio Pandeiros-Bonito de Minas/MG. MG. Biota, v. 2, p. 22-30, 2009b.

SALES, H. R.; SOUZA, S. C. A.; LUZ, G. R.; MORAIS-COSTA, F.; AMARAL, V. B.; SANTOS, R. M.; VELOSO, M. D. M.; NUNES, Y. R. F. Flora arbórea de uma Floresta Estacional Decidual na APA Estadual do rio Pandeiros, Januária/MG. MG. Biota, v. 2, p. 31-41, $2009 a$.

SANTOS, R. M.; VIEIRA, F. A.; FAGUNDES, M.; NUNES, Y. R. F.; GUSMÃO, E. Riqueza e similaridade florística de oito remanescentes florestais no Norte de Minas Gerais. Revista Árvore, Viçosa, v. 31, p. 135-144, 2007. Disponível em: <http://www.scielo.br/pdf/\%0D/rarv/v31n1/15.pdf>. Acesso em: 17 set. 2018.

SEVILHA, A. C.; SCARIOT, A.; NORONHA, S. Estado atual da representatividade de unidades de conservação em florestas estacionais deciduais no Brasil. In: 55 CONGRESSO NACIONAL DE BOTÂNICA. São Paulo. Anais ... São Paulo: Sociedade Brasileira de Botânica, p. 1-60, 2004.

SIMÕES, D.; GOBBI, N.; BATARCE, B. R. M. Mudanças sazonais na estrutura populacional em colônias de três espécies de vespas do gênero Mischocyttarus (Hymenoptera, Vespidae). Naturalia, v. 10, p. 89-105, 1985.

SOMAVILLA, A.; OLIVEIRA, M. L.; SILVEIRA, O. T. Identification guide for nests of social wasps (Hymenoptera: Vespidae: Polistinae) in Reserva Ducke, Manaus, Amazonas, Brazil. Revista Brasileira de Entomologia, v. 56, n. 4, p. 405-414, 2012.

SOUZA, M. M.; PREZOTO, F. Diversity of social wasps (Hymenoptera: Vespidae) in semideciduous forest and cerrado (Savanna) regions in Brazil. Sociobiology, v. 47, n. 1, p. 135-147, 2006. Disponível em: < https://www.researchgate.net/profile/Fabio_Prezoto/publication/289329514_Diversity_of_social_wasps_Hymenoptera_Vespidae_in_semideciduous_forest_and_cerrado_Savanna_regions_in_Brazil/links/58ebc1cbaca272bd2875f7dd/Diversity-of-social-wasps-Hymenoptera-Vespidae-in-semideciduous-forest-and-cerrado-Savanna-regions-in-Brazil.pdf>. Acesso em: 18 set. 2018.

SOUZA, M. M.; LADEIRA, T. E.; ASSIS, N. R. G. A.; ELPINO-CAMPOS, A.; CARVALHO, P.; LOUZADA, J. N. Ecologia de vespas sociais (Hymenoptera, Vespidae) no Campo Rupestre na Área de Proteção Ambiental, APA, São José, Tiradentes, MG. Biota, v. 3, n. 2, 15-30, 2010.

SOUZA, M. M.; PIRES, E. P.; ELPINO-CAMPOS, A.; LOUZADA, J. N. C. Nesting of social wasps (Hymenoptera: Vespidae) in a riparian forest of Rio das Mortes in Southeastern Brazil. Acta Scientiarum. Biological Sciences (Impresso), v. 36, p. 189-196, 2014. 
SOUZA, M. M.; PIRES, E. P.; PREZOTO, F. Seasonal richness and composition of social wasps (Hymenoptera, Vespidae) in áreas of Cerrado Biome in Barroso, Minas Gerais State, Brasil. Bioscience Journal, v. 30, p. 539-545, 2014.

TORRES, V. O.; MONTAGNA, T. S.; FERNANDES, W. D.; ANTONIALLI-JUNIOR, W. F. Colony cycle of the social wasp Mischocyttarus consimilis Zikán (Hymenoptera, Vespidae). Revista Brasileira de Entomologia, v. 55, p. 247-252, 2011. Disponível em: http://dx.doi.org/10.1590/S008556262011000200016 . Acesso em: 17 set. 2018.

VELOSO, H. P.; RANGEL-FILHO, A. L. R.; LIMA J. C. A. Classificação da vegetação brasileira, adaptada a um sistema universal. IBGE, Departamento de Recursos Naturais e Estudos Ambientais, Rio de Janeiro. p.123, 1991. Disponível em: <http://hm-jbb.ibict.br/bitstream/1/397/1/1991_classificacaovegetal_Velloso1991.pdf>. Acesso em: 17 set. 2018.

WENZEL, J. W. A generic key to the nests of hornets, yellowjackets, and paper wasps worldwide (Vespidae: Vespinae, Polistinae). American Museum Novitates, v. 3224, p. 1-39, 1998.

\section{Histórico editorial:}

Submetido em: 19/01/2017

Aceito em: 18/07/2017

Como citar:

$\underline{A B N T}$

FRANCISCO, G. S.; SOUZA, M. M. de; CLEMENTE, M.; BRUNISMANN, A. G. Substrato vegetal utilizado para nidificação de vespas sociais (Hymenoptera, Vespidae) em Floresta Decidual. Revista Agrogeoambiental, Pouso Alegre, v. 10, n. 3, p. 35-45, jul./set. DOI: http://dx.doi.org/10.18406/2316-1817v10n320181162

$\underline{\text { APA }}$

FRANCISCO, G. S., SOUZA, M. M. de, CLEMENTE, M. \& BRUNISMANN, A. G. (2018). Substrato vegetal utilizado para nidificação de vespas sociais (Hymenoptera, Vespidae) em Floresta Decidual. Revista Agrogeoambiental, 10 (3), 35-45. DOI: http://dx.doi.org/10.18406/2316-1817v10n320181162

ISO

FRANCISCO, G. S.; SOUZA, M. M. de; CLEMENTE, M. e BRUNISMANN, A. G. Substrato vegetal utilizado para nidificação de vespas sociais (Hymenoptera, Vespidae) em Floresta Decidual. Revista Agrogeoambiental, 2018, vol. 10, n. 3, pp. 35-45. Eissn 2316-1817. DOI: http://dx.doi.org/10.18406/2316-1817v10n320181162

\section{VANCOUVER}

Francisco GS, Souza MM de, Clemente M, Brunismann AG. Substrato vegetal utilizado para nidificação de vespas sociais (Hymenoptera, Vespidae) em Floresta Decidual. Rev agrogeoambiental. 2018. jul./set.; 10(3): 35-45. DOI: http://dx.doi.org/10.18406/2316-1817v10n320181162 


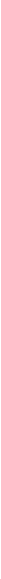

